\title{
Estrategias para reducir a un mínimo la transmisión nosocomial del sarampión ${ }^{1}$
}

\author{
R. J. Biellik ${ }^{2}$ y C. J. Clements ${ }^{3}$
}

RESUMEN Como resultado de la gran contagiosidad del sarampión antes de salir la erupción, la transmisión nosocomial seguirá siendo un peligro hasta que se erradique la enfermedad. No obstante, varias estrategias pueden reducir a un mínimo la transmisión nosocomial. Es imprescindible, por lo tanto, hacer que el personal de salud cobre mayor conciencia de que un individuo con sarampión puede llegar en cualquier momento a un servicio de salud y de que el peligro de transmisión del sarampión en el medio hospitalario existe de continuo. La presente revisión contiene dos grupos de recomendaciones: las que suelen ser aplicables a todos los países, y ciertas recomendaciones adicionales que pueden ser apropiadas solamente para países industrializados.

En la primera década del siglo XXI, es factible que el sarampión sea la próxima enfermedad -después de la viruela, la poliomielitis y la dracunculosis- en ser elegida para la erradicación mundial. No obstante, hasta no erradicarse, la enfermedad sigue siendo un peligro, especialmente para los niños que ingresan a los servicios de salud.

La contribución de la transmisión nosocomial a la incidencia de sarampión fluctúa notablemente, según la epidemiología del sarampión observada en una zona en particular. Numerosos estudios han demostrado que

1 Se publicó en inglés en el Bulletin of the World Health Organization, Vol. 75, No. 4, 1997, con el título "Strategies for minimizing nosocomial measles transmission". (c) Organización Mundial de la Salud, 1997.

2 Organización Mundial de la Salud, Harare, Zimbabwe.

3 Organización Mundial de la Salud, Programa Mundial de Vacunas e Inmunización, Programa Ampliado de Inmunización, Ginebra, Suiza. Las solicitudes de separatas en inglés deben dirigirse a este autor a la siguiente dirección postal: OMS, 1211 Ginebra 27, Suiza. la transmisión nosocomial es importante en los países industrializados y en zonas urbanas de países en desarro1lo. Sin embargo, este tipo de transmisión no contribuye de manera importante a la incidencia de sarampión en comunidades rurales de países en desarrollo, donde la cobertura de inmunización sigue siendo escasa o moderada y donde es limitado el acceso o el uso de los servicios de salud entre los miembros de la comunidad para enfermedades tales como el sarampión.

Hasta que se erradique el sarampión, será difícil eliminar su transmisión nosocomial debido a ciertos factores limitantes, entre ellos la gran contagiosidad de la enfermedad durante el período de incubación. No obstante, varias estrategias pueden reducir a un mínimo este tipo de transmisión. Por lo tanto es imprescindible hacer que los trabajadores de la salud cobren mayor conciencia de que un individuo con sarampión puede en cualquier momento llegar a un servicio de salud, con el fin de que el personal esté siempre atento al peligro de transmisión a personas no inmunes.

El sarampión es causado por uno de los agentes patógenos más virulentos para el ser humano y es, sin lugar a dudas, la más contagiosa de todas las enfermedades que pueden prevenirse mediante la vacunación. Esto se debe a la capacidad del virus de permanecer viable por períodos muy largos en forma de gotas finas que las personas infectadas expelen al toser (1-3). De ahí que la transmisión del sarampión se vea facilitada por la aglomeración de individuos susceptibles en espacios cerrados donde suelen quedar en el ambiente estos aerosoles infectantes.

Fuera del domicilio, los sitios de mayor riesgo son los centros educacionales, estadios deportivos, instituciones religiosas, orfelinatos, campamentos para refugiados y servicios de salud $(4,5)$. Estos últimos son los más alarmantes, puesto que acarrean el riesgo de que personas que ya podrían estar gravemente enfermas queden expuestas al sarampión. El uso de una 
jeringa estéril para cada inmunización o inyección terapéutica será garantía de que la infección no se propague por esta vía, pero tales precauciones no sirven para evitar la transmisión por partículas respiratorias en suspensión.

En este artículo se repasa la bibliografía sobre el tema, se cuantifica en lo posible la contribución de la transmisión nosocomial a la incidencia general del sarampión, y se ofrecen recomendaciones prácticas para idear estrategias orientadas a reducir a un mínimo la transmisión nosocomial.

\section{LA EPIDEMIOLOGÍA DEL SARAMPIÓN NOSOCOMIAL}

En comunidades donde circula el virus del sarampión, es probable que sea un agente patógeno común en los servicios de salud y una proporción importante de los individuos podrían infectarse al visitar uno de estos servicios. En un estudio sobre infecciones nosocomiales que se llevó a cabo de 1946 a 1947 en 26 salas pediátricas en 14 hospitales representativos ubicados por todo el Reino Unido se encontró que $12 \%$ de todas las infecciones nosocomiales eran casos de sarampión (6). Algo similar se encontró en niños de raza negra de estrato socioeconómico bajo que fueron internados en un hospital escuela importante de Sudáfrica en 1987 (7). Es posible que estos estudios hayan subestimado la fracción de infecciones nosocomiales debidas al sarampión, ya que la vigilancia después del egreso no siempre se llegó a poner en práctica.

La contribución relativa de la transmisión nosocomial a la incidencia total del sarampión varía de acuerdo con el patrón epidemiológico prevaleciente de la enfermedad (cuadro 1). El análisis de los datos publicados sugiere que está aumentando la proporción de casos adquiridos en el medio hospitalario en determinados contextos.

Se dispone de datos publicados sobre vigilancia de carácter nacional para los Estados Unidos de América de 1980 a 1990, período en el que la alta cobertura con vacunación había reducido la incidencia del sarampión a

CUADRO 1. Contribución relativa de la transmisión nosocomial a la incidencia de sarampión en países industrializados y en desarrollo

\begin{tabular}{|c|c|c|c|}
\hline País & Año & $\begin{array}{c}\text { \% de casos asociados } \\
\text { con transmisión } \\
\text { nosocomial }\end{array}$ & $\begin{array}{l}\text { Autor, año de } \\
\text { la publicación } \\
\text { (ref.) }\end{array}$ \\
\hline \multicolumn{4}{|l|}{ Países industrializados } \\
\hline Estados Unidos & 1980 & 0,4 & $C D C,{ }^{a} 1981(41)$ \\
\hline Estados Unidos & 1981 & 1,1 & Davis et al., 1986 (40) \\
\hline Estados Unidos & 1982 & 1,2 & Davis et al., 1986 (40) \\
\hline Estados Unidos & 1983 & 3,7 & Davis et al., 1986 (40) \\
\hline Estados Unidos & 1984 & 2,5 & Davis et al., 1986 (40) \\
\hline Estados Unidos & 1985 & 4,7 & CDC, $1986(42)$ \\
\hline Estados Unidos & 1987 & 5,8 & CDC, 1989 (43) \\
\hline Estados Unidos & 1989-90 & 9,0 & Gindler et al., 1992 (5) \\
\hline \multicolumn{4}{|c|}{$\begin{array}{l}\text { Brotes investigados (en la } \\
\text { comunidad) }\end{array}$} \\
\hline Estados Unidos (OK) & $1981-85$ & 27 & Istre et al., 1987 (11) \\
\hline Estados Unidos (CA) & 1983 & 29 & Dales et al., 1985 (10) \\
\hline Estados Unidos (HI) & 1984 & 15 & CDC, $1984(9)$ \\
\hline \multicolumn{4}{|c|}{$\begin{array}{l}\text { Brotes investigados (en centros } \\
\text { de salud) }\end{array}$} \\
\hline Francia & 1983-84 & 16 & Foulon et al., 1986 (38) \\
\hline Estados Unidos (MI) & 1985 & 57 & Sienko et al., 1987 (15) \\
\hline Estados Unidos (NM) & 1986 & 43 & CDC, 1987 (13) \\
\hline Sudáfrica & $1985-86$ & 25 & Reynolds et al., 1987 (14) \\
\hline Estados Unidos (MI) & 1990 & 48 & McGrath et al., 1992 (16) \\
\hline Estados Unidos (CA) & 1990 & 72 & Mason et at., 1993 (17) \\
\hline \multicolumn{4}{|l|}{ Casos y controles } \\
\hline Estados Unidos (CA) & 1988-89 & $23^{b}$ & Farizo et al., 1991 (18) \\
\hline Estados Unidos (TX) & $1988-89$ & $41^{\mathrm{b}}$ & Farizo et al., 1991 (18) \\
\hline \multicolumn{4}{|l|}{ Países en desarrollo } \\
\hline \multicolumn{4}{|c|}{$\begin{array}{l}\text { Brotes investigados (en la } \\
\text { comunidad) }\end{array}$} \\
\hline Brasil & 1983 & 21 & OPS, ${ }^{c} 1984(19)$ \\
\hline \multicolumn{4}{|c|}{$\begin{array}{l}\text { Brotes investigados (en centros de } \\
\text { salud) }\end{array}$} \\
\hline Camerún & 1975 & 35 & Guyer, 1976 (20) \\
\hline Mozambique & 1980 & 33 & Rabelo, 1982 (45) \\
\hline Guinea-Bissau & $1980-82$ & 16 & Aaby et al., 1985 (21) \\
\hline \multicolumn{4}{|l|}{ Casos y testigos } \\
\hline Côte d'Ivoire & 1985 & $71^{\mathrm{b}}$ & Klein-Zabban et al., 1987 (23) \\
\hline Burundi & 1988 & 32 & Chen, 1990 (24) \\
\hline
\end{tabular}

${ }^{a} \mathrm{CDC}=$ Centros para el Control y Prevención de Enfermedades.

${ }^{\text {b }}$ Más casos que controles habían visitado un centro de salud durante las 2 semanas previas al inicio de la erupción y la diferencia fue estadísticamente significativa (46 de 64 y 0 de $21 ; P<0,1$ ).

c OPS = Organización Panamericana de la Salud.

los niveles más bajos que se conocían hasta el momento y la mayoría de los casos notificados se asociaban con brotes aislados (8). Se disponía de información sobre la posible fuente de exposición en un promedio de alrededor de la mitad de los casos notificados. De ellos, menos de $10 \%$ se notificaron como habiéndose infectado en servicios de salud durante este período. No obstante, nuestra revisión de los datos publicados sugiere que la transmisión nosocomial en los Estados Unidos, como proporción de todos los casos transmitidos, aumentó 20 veces en forma casi lineal durante el mismo período. Aunque estos datos podrían estar sesgados (en una gran proporción de los casos no se había notificado la fuente de exposición), es poco pro- 
bable que el sesgo por sí solo explique la tendencia tan marcada en la proporción de casos transmitidos dentro de hospitales.

Datos tomados de investigaciones sobre brotes de sarampión en tres países industrializados (Francia, Sudáfrica y Estados Unidos) muestran que la proporción de casos en que la exposición tuvo lugar en un servicio de salud varía de 15 a $72 \%$. Esta proporción tendió a ser menor en estudios de base comunitaria (9-11) que en estudios de brotes o casos hospitalizados en servicios de salud de zonas urbanas (12-17). Esto coincide con lo anticipado, ya que los estudios efectuados en servicios tienden a sobrerrepresentar a aquellos miembros de la comunidad que usan más los servicios de salud y, por lo tanto, tienden a sobreestimar la tasa de infección en la comunidad. Estudios de casos y controles efectuados en los Estados Unidos sobre la transmisión del sarampión en Los Ángeles y Houston han mostrado que el haber sido atendido en la sala de emergencia de un hospital 10 a 18 días antes del inicio de la erupción sarampionosa fue un factor de riesgo relevante (18).

Según datos obtenidos mediante investigaciones sobre brotes de sarampión en seis países en desarrollo (Brasil, Burundi, Camerún, Côte d'Ivoire, Guinea-Bissau y Mozambique), la proporción de casos de sarampión en los que la exposición tuvo lugar en servicios de salud fluctuó entre 21 y $71 \%$. La proporción de casos asociados con la transmisión nosocomial por lo general fue similar en un estudio de tipo comunitario (19) en el Brasil y en estudios efectuados en servicios o pacientes hospitalizados en zonas urbanas $(20,21)$. Un estudio de casos y controles que se llevó a cabo en Abiyán mostró que el haber visitado la sala de emergencia de un hospital en las últimas 2 semanas o menos (que es el período de incubación aproximado del sarampión) fue un factor de riesgo significativo para contraer el sarampión $(22,23)$. No obstante, en un estudio en una zona rural de Burundi no se halló ninguna diferencia en la proporción de casos y controles que habían visitado un centro de salud en el último mes (24). Según un estudio comunitario en Burundi, solamente $1 \%$ de las personas con sarampión habían acudido a un centro de salud en el último mes.

En resumen, estos estudios sugieren que la transmisión nosocomial es importante en países industrializados $\mathrm{y}$ en zonas urbanas de países en desarro1lo. Además, datos procedentes de los Estados Unidos indican que la contribución relativa de la transmisión nosocomial aumentará a medida que se alcancen tasas elevadas de cobertura con vacunación en más países (25) y que se reduzca la importancia del sarampión endémico (el que no obedece a un brote). Estos estudios también sugieren que la transmisión nosocomial no contribuye de manera importante a la incidencia de sarampión en comunidades rurales de países en desarrollo donde la cobertura con vacunación sigue siendo baja o moderada y donde es limitado el acceso y uso de servicios de salud por parte de los miembros de la comunidad para enfermedades como el sarampión.

\section{FACTORES QUE INFLUYEN EN EL CONTROL DEL SARAMPIÓN NOSOCOMIAL}

\section{Características de la transmisión}

El sarampión es una enfermedad aguda y muy contagiosa que se propaga por contacto directo con gotitas infecciosas (26). La inmunidad conferida por la enfermedad dura toda la vida como también, para fines prácticos, la seroconversión después de una inmunización eficaz.

El virus se excreta en la nasofaringe de personas enfermas y se expele en gotitas suspendidas en el aire al toser. Varios estudios han demostrado que la infección puede producirse sin que haya contacto cara a cara con un individuo contagioso. La transmisión del sarampión se ha documentado aun cuando la persona contagiosa ha abandonado la habitación hasta 2 horas antes de la llegada de las que poste- riormente han contraído la infección $(1,3,9,13,15,27-29)$. En una escuela secundaria de los Estados Unidos, un niño que tenía una tos muy fuerte causó una rápida epidemia de sarampión de una sola generación en 69 niños no inmunes, muchos de los cuales no habían tenido contacto directo con el caso índice pero sí habían pasado por los mismos corredores entre clases (29).

En vista de que los pacientes con sarampión se encuentran en estado contagioso de 3 a 5 días antes de que empiece la erupción (1 a 2 días antes del inicio de la fiebre y otros síntomas), puede resultar difícil identificar a los individuos infecciosos, muchos de los cuales tienen síntomas atípicos. Tales pacientes facilitan la transmisión nosocomial y dificultan su control.

\section{Edad de los pacientes y gravedad de la enfermedad}

La transmisión del sarampión se ve facilitada por el contacto cercano entre seres humanos y el hacinamiento (30), tal como se observan en centros de salud muy activos. También existe un mayor riesgo de sarampión grave en condiciones de hacinamiento como las que suele haber en países en desarrollo (31-36). De hecho, el hacinamiento (que sirve como sustituto [proxy] de la intensidad de la dosis vírica) es más importante que el estado nutricional (que es sustituto de la resistencia del huésped) como factor que determina la gravedad de la enfermedad $(33,35)$. Otros estudios han mostrado que las tasas de complicación y de letalidad fueron notablemente más altas, y que los tiempos de recuperación más largos, en menores de un año y niños pequeños que contrajeron el sarampión dentro de un servicio hospitalario, al compararse con los que lo contrajeron en el seno de la comunidad (14, 37-39).

En los Estados Unidos de 1983 a 1984, 20\% de los casos de sarampión se produjeron en menores de un año y niños demasiado pequeños para ser candidatos a la vacunación, mientras que no podían ser candidatos $59 \%$ de 
los que contrajeron el sarampión en un servicio de salud (40). Los menores de un año y los niños pequeños pueden estar en mayor riesgo de contagio nosocomial porque acuden con mayor frecuencia a los centros de salud para fines tales como la inmunización y la atención del niño sano.

\section{Características de los servicios de salud}

Los servicios de salud desempeñan un papel importante en la transmisión del sarampión, no solo en su interior sino en la comunidad en general y tanto en los países industrializados $(1,3,5,9,11,13-18,28,38,40-43)$ como en los países en desarrollo $(7,19-21$, $23,24,38,44,45)$. Un estudio retrospectivo de 5 años que se realizó en los Estados Unidos a principios de los años ochenta abarcó todo tipo de servicios de salud; las infecciones sarampionosas nosocomiales se encontraron en mayores proporciones en los hospitales $(47 \%)$, consultorios médicos (35\%) y clínicas ambulatorias (15\%) (40). Durante brotes de sarampión de alcance comunitario en dos ciudades estadounidenses, el haber visitado una sala de emergencias hospitalaria en los 10 a 18 días anteriores a la aparición de la erupción sarampionosa fue un factor de riesgo significativo (19). La transmisión del sarampión también se produjo en farmacias, centros de emergencia no hospitalarios, un centro de rehabilitación para personas farmacodependientes y un laboratorio. Un total de $45 \%$ de los casos de sarampión que fueron evaluados en otro estudio estadounidense obedecieron directa o indirectamente a la exposición en la sala de espera de un servicio médico (11). En países en desarrollo, las clínicas de atención materna e infantil se han visto involucradas en la propagación nosocomial del sarampión (20, 38). Según estudios sobre brotes de sarampión en los Estados Unidos, la humedad relativa baja y la falta de circulación de aire fresco en las salas de espera facilitan la transmisión del sarampión (3).

\section{El papel del personal de salud}

Si bien la mayor parte de los brotes de sarampión en un medio hospitalario ocurren en pacientes y visitantes que carecen de inmunidad, trabajadores de salud que no están inmunes a menudo se ven afectados, sobre todo en países donde el sarampión no ha sido endémico por muchos años (46-51). El estudio retrospectivo de 5 años mencionado anteriormente que se efectuó en Estados Unidos a principios de los años ochenta reveló que, de los casos de sarampión transmitidos dentro de hospitales, 50\% habían sido transmitidos de un paciente a otro, $37 \%$ de un paciente a un miembro del personal, $8 \%$ de un paciente a un visitante, 3\% de un miembro del personal a otro y $2 \%$ de un miembro del personal a un paciente (40). Los empleados que contrajeron la enfermedad con mayor frecuencia fueron los que tenían contacto con pacientes o con muestras de laboratorio, entre ellos miembros del personal de enfermería, médicos, técnicos de laboratorio y oficinistas o recepcionistas. A diferencia de lo que se observa en países industrializados, muchos trabajadores de salud en países en desarrollo crecieron antes de que hubiera amplia disponibilidad de la vacuna antisarampionosa. Por lo tanto, es más probable que estén inmunizados por exposición (usualmente en la infancia) y por consiguiente no contribuyen a la transmisión nosocomial.

\section{LA PREVENCIÓN POR MEDIO DE LA VACUNACIÓN}

Si todos los menores de un año y niños adquirieran la inmunidad contra el sarampión antes de ser expuestos a una persona contagiosa en un contexto médico, no habría transmisión nosocomial. No obstante, la vacunación reglamentaria de los niños de 9 meses o mayores no protege a los lactantes susceptibles menores de esa edad. Tampoco estarán protegidas las personas que reciben la vacuna antisarampionosa pero que no responden con títu- los de anticuerpos protectores. Por añadidura, la determinación del estado de los lactantes y niños menores de un año con respecto a la vacunación, especialmente en países en desarrollo, puede verse dificultada por la poca frecuencia con que se conserva la tarjeta de vacunación. La prevención del sarampión nosocomial por medio de la vacunación previa a la exposición exige, por lo tanto, estrategias de vacunación adicionales.

Varios estudios han indicado que la vacuna antisarampionosa es eficaz para prevenir la presentación del cuadro clínico en individuos expuestos si la vacunación se lleva a cabo 72 horas o menos después de la exposición (52). La tasa de protección fluctuó de 68 a 100\%. En cuatro estudios sobre la exposición dentro de domicilios o instituciones (incluida la de tipo nosocomial) se notificaron tasas de protección de $94 \%$ o más (53-56). Sin embargo, aún no se ha estudiado la eficacia de la profilaxis después de la exposición en niños menores de 1 año y especialmente en los menores de 9 meses. Estos resultados respaldan, no obstante, las recomendaciones al efecto de que los individuos deben vacunarse contra el sarampión en un lapso de 72 horas después de la exposición (26).

\section{RECOMENDACIONES PARA EL CONTROL}

Dado que el sarampión es muy contagioso durante la fase preceptiva, será sumamente difícil eliminar su transmisión nosocomial hasta que se erradique la enfermedad misma. No obstante, numerosas estrategias pueden minimizar la propagación nosocomial. Las siguientes recomendaciones se dividen entre las que pueden aplicarse a todos los países y las que pueden ser apropiadas solo para países industrializados.

\section{Medidas aplicables para todos los países}

Mantener alto el grado de conciencia del personal de salud. Es imprescindi- 
ble hacer más conscientes a los miembros del personal de los servicios de salud de que una persona con sarampión puede entrar al servicio en cualquier momento y de que en él existe un riesgo continuo de que se transmita el sarampión a personas que carecen de inmunidad.

Mantener alta la cobertura en la comunidad. La acumulación de individuos susceptibles en la comunidad se reducirá a un mínimo si se mantienen altas las tasas de cobertura con la vacuna antisarampionosa. Se han descrito varias estrategias para alcanzar una alta cobertura (25). Podría ser necesaria una combinación, incluida la vacunación reglamentaria, para grupos o zonas en alto riesgo, y campañas en masa.

En vista de que no hay prácticamente ninguna contraindicación para la vacuna antisarampionosa, esta debe administrarse sin importar el estado de salud del paciente. La vacunación antisarampionosa reviste especial importancia en el caso de niños desnutridos o con enfermedades crónicas, que tienen un mayor riesgo de sufrir complicaciones producidas por el sarampión. Se exceptúan de esta recomendación los niños que se encuentran tan enfermos al ingreso que están en grave peligro de morir. Aunque la administración de la vacuna antisarampionosa no es peligrosa en estos casos, los padres podrían atribuir equivocadamente la muerte del niño a la vacunación.

Reducir la edad para la vacunación entre brotes. La OMS recomienda bajar la edad para la administración de la vacuna contra el sarampión a 6 meses durante brotes en que se espere una alta tasa de ataque. La vacuna debe administrarse en cualquier momento a partir de los 6 meses y, en niños vacunados entre los 6 y los 9 meses de edad, debe administrarse una segunda dosis tan pronto sea posible después de los 9 meses, siempre que hayan transcurrido por lo menos 4 semanas desde la última dosis de va- cuna. La segunda dosis después de la edad de 9 meses es importante, ya que la respuesta serológica a la vacuna administrada antes de los 9 meses puede ser muy inferior y resultar en niveles de protección más bajos. Los padres deben recibir instrucciones adecuadas cuando se administra una dosis temprana. (La recomendación sobre la revacunación de niños vacunados antes de los 9 meses de edad también se aplica a las secciones siguientes.)

Eliminar la pérdida de oportunidades para vacunar. Debe eliminarse la pérdida de oportunidades para vacunar mediante la determinación reglamentaria del estado de vacunación de todos los niños que acudan a cualquier servicio de salud sin importar el motivo. Esto debe ponerse en práctica en todas las clínicas de inmunización, consultorios médicos, clínicas públicas y privadas, centros de salud y salas de emergencia y de atención ambulatoria dentro de hospitales. Aun los niños con antecedentes de sarampión deben vacunarse, puesto que los médicos, trabajadores de salud, madres y otros prestadores de atención a menudo confunden otras enfermedades febriles y eruptivas con el sarampión. Tampoco basta con tener antecedentes verbales de vacunación previa para contraindicar la vacuna. Para este fin solamente debe aceptarse documentación en forma de una tarjeta de vacunación.

En países en desarrollo, donde a menudo faltan las tarjetas de vacunación y las fichas médicas, deben evaluarse las condiciones locales a la hora de definir los criterios para la administración de la vacuna antisarampionosa a niños cuyo estado con respecto a la vacuna no puede determinarse. Cuando así lo indique el análisis epidemiológico, incluida la evaluación de la tasa de letalidad, la respuesta a los brotes de sarampión podría abarcar la vacunación de todos los niños entre los 6 ó 9 meses de edad y los 9 años, independientemente de los antecedentes de vacunación antisarampionosa. Cuando no hay un brote, se recomienda que todos los niños entre las edades de 9 meses y 2 años, como mínimo, que carezcan de documentos que confirmen que han sido vacunados reciban la vacuna antisarampionosa.

Se dispone de un método sencillo para determinar la pérdida de oportunidades para vacunar (57). Debe procurarse de forma sistemática eliminar todas las oportunidades perdidas. Cualquier niño que sea candidato para la vacuna antisarampionosa ( $u$ otros antígenos, y cualquier mujer que sea candidata para el toxoide tetánico) debe recibir cualquier dosis o dosis que falten antes de abandonar el servicio de salud. Si una persona sin inmunidad se expone al sarampión por contacto directo o indirecto con una persona contagiosa, por lo general la vacuna antisarampionosa le conferirá inmunidad si se administra en el transcurso de las 72 horas posteriores a la exposición.

Garantizar la vacunación de pacientes hospitalizados. Debe investigarse con rigor el estado de todos los niños ingresados a un hospital con respecto a la vacunación. La OMS recomienda que, en países en desarrollo, se administre una dosis de vacuna antisarampionosa a todos los niños entre las edades de 6 meses y 9 años (ajustando el recorrido de edades según las condiciones en la localidad) cuando se ingresen en el hospital, aunque exista documentación de que ya han sido vacunados. En la mayor parte de los países industrializados, solamente los pacientes sin documentación de haber sido vacunados tienen que vacunarse al ingresar.

En el mejor de los casos, la vacunación se asocia con una eficacia de 80 a $90 \%$ en países en desarrollo $(19,58$ 61). De ahí que una dosis adicional de vacuna antes del ingreso al hospital pueda reducir notablemente la factibilidad de que se produzca la transmisión nosocomial del sarampión.

También se debe determinar el estado de los niños hospitalizados con respecto a la vacuna antes de darles de alta del hospital. La vacunación de los niños sin documentación de que han sido vacunados reduce las probabili- 
dades de que el niño regrese a su casa mientras está incubando una infección sarampionosa nosocomial. El no cumplir con esta medida podría llevar a la infección de otros niños en la comunidad.

Las personas sin inmunidad que tengan más de 6 meses de edad y que hayan estado expuestas a pacientes con sarampión dentro del hospital, tales como los pacientes que están en una misma sala y que tienen las mismas visitas, deben recibir una dosis de vacuna contra el sarampión, siempre que sea posible en un lapso de 72 horas después de la exposición. En el caso de pacientes sin deficiencia inmunitaria, el uso de gammaglobulina hiperinmune contra el sarampión (IMGG) puede ser menos eficaz y más caro que la vacuna antisarampionosa.

Aislar a las personas que tengan fiebre y erupción. Para reducir las probabilidades de sufrir exposición al sarampión, las personas con fiebre y erupción deben de preferencia abstenerse de entrar en las salas de espera comunes que suele haber en los servicios de salud. Siempre que sea factible, a estos pacientes se les debe poner una mascarilla y llevar directamente a una sala reservada especialmente para pacientes con enfermedades sujetas a aislamiento respiratorio. De ser posible, las áreas de espera y de tratamiento deben estar bien ventiladas $y$, en la medida de las posibilidades, deben tomarse precauciones para que los niños enfermos y sanos no usen la misma habitación consecutivamente y no sean manejados por el mismo personal para la toma del peso, los exámenes físicos, las vacunaciones $\mathrm{u}$ otras consultas, ya que esto bien podría servir de medio para la transmisión del sarampión. En lugares donde es común que las mujeres sean alfabetas, se puede montar un letrero a la entrada del servicio para advertir a los padres $u$ otros responsables que acompañen a un niño con fiebre y erupción que espere afuera y que le pida a otro usuario que le avise al personal que ha llegado un niño enfermo.

A fin de reducir la gravedad del sarampión y el riesgo de complicaciones posteriores, deben administrarse suplementos de vitamina A (200 000 UI dos días consecutivos a niños mayores de 1 año) a todos los niños hospitalizados en quienes se sospeche sarampión (62).

Informar a las autoridades de salud. El sarampión es una enfermedad notificable en casi todos los países. Los casos de sarampión deben notificarse con prontitud a las autoridades sanitarias del distrito en conformidad con las prácticas establecidas localmente. Los casos de sarampión nosocomial deben también notificarse de inmediato a las autoridades dedicadas al control de infecciones para que los investiguen $y$ monten una respuesta, que podría ser una intensificación de las medidas para el aislamiento de niños enfermos.

\section{Medidas de control para países industrializados}

Garantizar la vacunación del personal de los servicios de salud. Todos los miembros del personal de los servicios de salud deben estar inmunizados contra el sarampión. La mayoría de los adultos en países en desarrollo ya tendrán inmunidad natural. Especialmente en países industrializados, donde puede haber adultos sin inmunidad, cualquier miembro del personal que no tenga documentación de que ya ha sido vacunado contra el sarampión o de que tiene títulos adecuados de anticuerpos contra la enfermedad en el momento de ser contratado debe recibir la vacuna antisarampionosa, después de determinarse si tiene contraindicaciones, tales como el embarazo o la supresión inmunitaria.

Administrar gammaglobulina a pacientes determinados. Ya que existe el riesgo de una viremia fulminante, las vacunas a base de virus vivos, como la del sarampión, están contraindicadas en personas con trastornos congénitos de la función inmunitaria o en las que reciben un tratamiento inmunosupresor. Por lo tanto, los individuos inmunocomprometidos que entran en contacto con pacientes de sarampión deben recibir IMGG tan pronto sea posible después de la exposición. No obstante, las personas seropositivas al virus de la inmunodeficiencia humana (VIH), o aquellas en quienes se sospecha o se ha confirmado la presencia del síndrome de inmunodeficiencia adquirida (sida), pueden recibir la vacuna antisarampionosa a base de virus vivos (53).

\section{CONCLUSIONES}

Hasta que se erradique el sarampión, su transmisión nosocomial sigue siendo un peligro. Reducir mediante la inmunización la cantidad de virus que circula en la comunidad sigue siendo la estrategia preferida para combatir el sarampión. No obstante, hay medidas adicionales que ayudarán a reducir la transmisión del sarampión en el ámbito de los servicios de atención de salud. Entre estas estrategias figura la determinación del estado de vacunación al ingreso y egreso, la vacunación del personal y el aislamiento de pacientes con fiebre y erupción, especialmente en situaciones de epidemia. Estrategias tales como la identificación de posibles casos de sarampión en las áreas de espera de hospitales y centros de salud y modificaciones estructurales del flujo de las corrientes de aire pueden ser beneficiosas, pero son más difíciles de poner en práctica y raras veces se han sometido a prueba.

Agradecimiento. Los autores agradecen los comentarios y las sugerencias en torno al manuscrito de F. Cutts, J. Gindler y J.-M. Olivé. 


\section{REFERENCIAS}

1. Bloch AB. Measles in a private physician's office. En: Proceedings of the $16^{\text {th }}$ Immunization Conference. Atlanta: Centers for Disease Control and Prevention; 1981. pp. 59-63.

2. Langmuir AD. Changing concepts of airborne infection of acute contagious diseases: A reconsideration of classical epidemiologic theories. Ann N Y Acad Sci 1980;353: 35-44.

3. Remington PL, et al. Airborne transmission of measles in a physician's office. JAMA 1985; 253:1574-1577.

4. Markowitz LE, et al. Patterns of transmission in measles outbreaks in the United States, 1985-86. N Engl J Med 1989;320:75-81.

5. Gindler JS, et al. Epidemiology of measles in the United States in 1989 and 1990. Pediatr Infect Dis J 1992;8:616-619.

6. Watkins AG, Lewis-Faning E. Incidence of cross-infection in children's wards. Br Med J 1949;2:616-619.

7. Cotton MF, et al. Nosocomial infections in black South African children. Pediatr Infect Dis I 1989:8:676-683.

8. World Health Organization. Measles control in the 1990s: Principles for the next decade. Geneva: WHO; 1990. (Documento inédito WHO/EPI/GEN/90.2)

9. Centers for Disease Control and Prevention. Measles — Hawaii. MMWR 1984;33(50):702, 707-711.

10. Dales LG, Kizer KW. Measles transmission in medical facilities. West J Med 1985;142: 415-416.

11. Istre GR, et al. Measles spread in medical settings: An important focus of disease transmission? Pediatr 1987;79:356-358.

12. Foulon $G$, et al. Preventing the spread of measles in children's clinics. Lancet 1983;2: 1498-1499.

13. Centers for Disease Control and Prevention. Measles transmitted in a medical office building - New Mexico, 1986. MMWR 1987; 36(2):25-27.

14. Reynolds LG, Klein M. The hospital as a vector of measles in the community. South African Med J 1987;71:637-638.

15. Sienko DG, et al. A measles outbreak at university medical settings involving health care providers. Am J Public Health 1987;77: 1222-1224.

16. McGrath D, et al. Analysis of a measles outbreak in Kent County, Michigan in 1990. Pediatr Infect Dis J 1992;11:385-389.

17. Mason $\mathrm{WH}$, et al. Epidemic measles in the post vaccine era: Evaluation of epidemiology, clinical presentation and complications during an urban outbreak. Pediatr Infect Dis J 1993;12:42-48

18. Farizo KM, et al. Paediatric emergency room visits: A risk factor for acquiring measles. Paediatrics 1991;87:74-79.

19. Pan American Health Organization. Investigation of a measles outbreak in Brazil's Federal District. Bull Pan Am Health Organ 1984;18: 397-400.

20. Guyer B. Transmission de la rougeole'a Youandé en 1975. Med Trop 1976;36:451-454.

21. Aaby $\mathrm{P}$, et al. Introduction of measles into a highly immunized West African community: The role of health care institutions. J Epidemiol Comm Health 1985;39:113-116.
22. World Health Organization. Expanded Programme on Immunization: Nosocomial measles. Wkly Epidemiol Rec 1986;61(44): 338-340.

23. Klein-Zabban ML, et al. Fréquence des rougeoles nosocomiales dans un centre de protection maternelle et infantile d'Abidjan. Bull World Health Organ 1987;65:197-201.

24. Chen RT. Report on epidemiologic studies conducted for the 1988-89 measles outbreak. Muyinga Health Sector, Burundi. Atlanta: Centers for Disease Control and Prevention; 1990.

25. World Health Organization. Revised plan of action for global measles control. Geneva: WHO; 1993. (Documento inédito EPI/GAG/ 93?WP.10)

26. American Academy of Pediatrics. Report of the Committee on Infectious Diseases. Elk Grove Village, Illinois: AAP; 1991.

27. Centers for Disease Control and Prevention Interstate importation of measles following transmission in an airport - California, Washington, 1982. MMWR 1983:32(16):210, 215-216.

28. Centers for Disease Control and Prevention. Imported measles with subsequent airborne transmission in a pediatrician's office Michigan. MMWR 1983;32(31):401-403.

29. Chen RT, et al. An explosive point-source measles outbreak in a highly vaccinated population. J Epidemiol 1989;129:173-182.

30. Greenwood M. Epidemics and crowd-diseases: Measles. Rev Infect Dis 1988;10:492-499.

31. Sharma RS, et al. An epidemiological investigation of a measles outbreak in AlwarRajastan. J Commun Dis 1984;16:299-302.

32. Aaby $\mathrm{P}$, et al. Overcrowding and intensive exposure as determinants of measles mortality. Am J Epidemiol 1984;120:49-63.

33. Aaby $\mathrm{P}$, et al. High measles mortality in infancy related to intensity of exposure. J Pediatr 1986;109:40-44.

34. Aaby $\mathrm{P}$, et al. Further community studies on the role of overcrowding and intensive exposure on measles mortality. Rev Infect Dis 1988, 10:474-491.

35. Lamb WH. Epidemic measles in a highly immunized rural West African (Gambian) village. Rev Infect Dis 1988:10:457-462.

36. Garenne M, Aaby P. Pattern of exposure and measles mortality in Senegal. I Infect Dis 1990;161:1088-1094

37. Glyn-Jones R. Measles vaccine and gamma globulin in the prevention of cross infection with measles in an acute paediatric ward. Cent Afr J Med 1972;18:4-9.

38. Foulon $\mathrm{G}$, et al. Transmission and severity of measles acquired in medical settings. JAMA 1986;256:1135-1136.

39. Hussey G, Simpson J. Nosocomial bacteraemia in measles. Pediatr Infect Dis J 1990; 9:715-717

40. Davis RM, et al. Transmission of measles in medical settings: 1980 through 1984. JAMA 1986;255:1295-1298.

41. Centers for Disease Control and Prevention Measles in medical settings. MMWR 1981; 30(11):125-126.

42. Centers for Disease Control and Prevention. Measles — United States, 1985. MMWR 1986; 35(22):366-370
43. Centers for Disease Control and Prevention. Measles - United States, 1988. MMWR 1989; 38(35):601-605

44. Gao JP, Malison MD. The epidemiology of a measles outbreak on a remote offshore island near Taiwan. Int J Epidemiol 1988;17:894-898.

45. Rabelo AL. [Observaciones sobre un brote de sarampión en un área periférica de la ciudad de Maputo]. Rev Med Moçambique 1982;1(1): 9-13. (En portugués)

46. Anguidanos R, Mascola L, Frederick P. A survey of hospital infection control policies and employee measles cases during Los Angeles County's measles epidemic, 1987 to 1989. Am J Infect Control 1992;20:301-304.

47. Gurevich I, Barganza RA, Cunha BA. Measles: Lessons from an outbreak. Am I Infect Control 1992;20:319-325.

48. Navarrete-Navarro $S$, et al. [El sarampión nosocomial: propuestas para el control en hospitales]. Bol Med Hosp Infant Mex 1990;47: 495-499. (En español).

49. Rivera ME, et al. Nosocomial measles infection in a pediatric hospital during a community-wide epidemic. J Pediatr 1991;119: 183-186.

50. Schwarcz S, McCaw B, Fukushima P. Prevalence of measles susceptibility in hospital staff. Arch Intern Med 1992:152:1481-1483.

51. Subbrao EK, Amin S, Kumar ML. Pre-vaccination serologic screening for measles in health care workers. J Infect Dis 1991;163: 876-878.

52. World Health Organization. Post-exposure prophylaxis. Geneva: WHO; 1993. (Documento inédito EPI/TECHCOM/WP/93.19).

53. Watson GI. Protection after exposure to measles by attenuated vaccine without gamma-globulin. Br Med J 1963;1:860-861.

54. Berkovich A, Starr S. Use of live-measlesvirus vaccine to abort an expected outbreak of measles within a closed population. New Engl J Med 1963;269:75-77.

55. Ruskanen O, Salmi TT, Halonen P. Measles vaccination after exposure to natural measles. J Pediatr 1978;93:43-45.

56. Addis DG, Berg JL, Davis JP. Revaccination of previously vaccinated siblings of children with measles during an outbreak. J Infect Dis 1988;157:610-611.

57. World Health Organization Protocol for the assessment of missed opportunities for immunization. Geneva: WHO; 1996. (Documento inédito WHO/EPI/GEN/86.6).

58. Hull HF, Williams PJ, Oldfield F. Measles mortality and vaccine efficacy in rural West Africa. Lancet 1983;1:972-975.

59. World Health Organization. Expanded Programme on Immunization: Measles vaccine efficacy. Wkly Epidemiol Rec 1984;59(17): 127-128.

60. Dabis F, et al. The epidemiology of measles in a partially vaccinated population in an African city: Implications for immunization programs. Am I Epidemiol 1988;127:171-178.

61. Thapa AB. Measles outbreak in a partially vaccinated population and measles vaccine efficacy, South Lalitpur District, Nepal, 1990. J Nepal Med Assn 1992;30:154-158.

62. Clements CJ, von Reyn CF, Mann JM. HIV infections and routine childhood immunization. Bull World Health Organ 1987;65:905-911. 
ABSTRACT Because of the highly contagious nature of measles before the onset of rash, nosocomial transmission will remain a threat until the disease is eradicated. However, a number of strategies can minimize its nosocomial spread. It is therefore vital to maximize awareness among health care staff that an individual with measles can enter a health facility at any time and that

Strategies for minimizing nosocomial measles transmission a continual risk of the nosocomial transmission of measles exists. The present review makes two groups of recommendations: those which are generally applicable to all countries, and certain additional recommendations which may be suitable only for industrialized countries.

\title{
Escuela de verano internacional
}

\author{
Fechas: $\quad 4$ a 22 de enero de 1999 \\ Lugar: $\quad$ Santiago, Chile
}

La Escuela de Salud Pública de la Universidad de Chile invita a todos los investigadores y otros profesionales del área de la salud a participar en la Escuela de verano internacional que organiza junto con la Escuela de Salud Pública de la Universidad de Carolina del Norte (EUA) y la Organización Panamericana de la Salud. La escuela de verano está orientada a impartir los principios básicos de la investigación epidemiológica y de los diseños de ensayos clínicos. Al final de los cursos, los participantes tendrán conocimientos básicos de bioestadística y metodología epidemiológica y una apreciación del diseño, la ejecución y el análisis de los ensayos clínicos, además de haber profundizado en ramos específicos de la epidemiología.

Los cursos que se ofrecen son los siguientes: introducción a la bioestadística; metodología epidemiológica; diseños de ensayos clínicos; análisis de datos en ensayos clínicos; epidemiología de enfermedades cardiovasculares; epidemiología ambiental; y técnicas avanzadas de análisis epidemiológico. La inscripción estará abierta hasta el 31 de diciembre de 1998, pero los que se inscriban antes del 30 de noviembre, recibirán una rebaja. La matrícula cuesta US\$ 200 por un curso, $\$ 385$ por dos, $\$ 560$ por tres, $\$ 725$ por cuatro, $\$ 880$ por cinco y $\$ 1025$ por seis.

\section{Información:}

Oficina de Relaciones Públicas, Escuela de Salud Pública

Casilla 70012, Correo 7, Santiago, Chile

Fax: (56) (2) 737 7121; Teléfonos: (56) (2) 678 6432/6150/6159

Correo electrónico: mcumsill@machi.med.uchile.cl 